\title{
DER BEITRAG DES THEODOROS STUDITES ZUR BYZANTINISCHEN HYMNOGRAPHIE
}

Eine der bedeutendsten Persönlichkeiten des kirchlichen und kirchenpolitischen Lebens in Byzanz war an der Wende vom 8. zum 9. Jahrhundert der Mönch Theodoros Studites (759-826) ${ }^{1}$. Er entstammte einer vornehmen Familie, trat im Jahr 780 mit seinem Bruder Joseph (762-832), dem späteren Erzbischof von Thessalonike, in das von seinem Onkel Platon geleitete Kloster Sakkudion in Bithynien ein. Im Jahr 794 wurde Theodoros Abt dieses Klosters. Wegen der drohenden Arabergefahr übersiedelte die Klostergemeinde im Jahr 798 in das halb verlassene Studiu-Kloster in Konstantinopel $^{2}$. Unter Theodoros erreichte dieses Kloster eine Blüte, die für das gesamte Mönchtum von entscheidender Bedeutung werden sollte. Theodoros setzte sich für das koinobitische Ideal nach dem Vorbild Basileios des Großen ein, für das Leben in der Klostergemeinschaft. Das StudiuKloster wurde zum Zentrum klösterlicher Reform und wirkte in weiterer Folge bis in den slavischen Raum. In der Auseinandersetzung mit dem Ikonoklasmus wirkte das Studiu-Kloster als spiritueller Mittelpunkt der Bilderverehrung.

Obwohl vom Studiu-Kloster aus der Zeit des Theodoros kein liturgisches Typikon erhalten ist ${ }^{3}$, dürfen wir doch annehmen, dass in dieses Dokument wesentliche Elemente des palästinensischen Ritus, vor allem des

1 Die Vita des Theodoros wurde von einem Mönch des Studiu-Klosters, Michael Monachos, verfasst: PG 99, 233-388. Theodoros wird am 11. November gefeiert. Vgl. H. Delehaye, Synaxarium Ecclesiae Constantinopolitanae e codice Sirmondiano nunc Berolinensi adiectis synaxariis selectis. Brüssel 1902 (Nachdruck Brüssel 1954), 214-216. Das Kon-

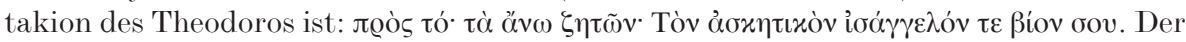
Festtag des Theodoros mit seinem Kontakion findet sich in Kondakarien-Notation, in kirchenslavischer Sprache, im Blagověščenskij Kondakar', ed. von A. DostaL - H. Rothe, unter Mitarbeit von E. Trapp, Der altrussische Kondakar' (Bausteine zur Geschichte der Literatur bei den Slawen 8,2). Gießen 1976, 17a-b.

${ }^{2}$ Das Studiu-Kloster geht auf eine Gründung des Jahres 462 zurück.

${ }^{3}$ Ein Fragment eines Klostertypikons des Codex Athos Vatopedi 322 (956) des 13./14. Jahrhunderts ist erhalten bei: A. DмiтRievskiJ, Opisanie liturgičeskich rukopisej chranjaščichsja v bibliotekach pravoslavnago Vostoka I. Tvлıxó. Kiev 1895, 224-238. 
Sabas-Klosters, übernommen worden waren, erweitert um eine Hymnographie, die im Einflussbereich Konstantinopels entstanden war bzw. entstand.

Theodoros verfasste, entsprechend der palästinensischen Tradition, Kanones $^{4}$ und Stichera ${ }^{5}$, er übernahm aber auch die vor allem im Raum Konstantinopel entstandene hymnische Form, das Kontakion.

Der Einfluss des Theodoros Studites auf die byzantinische Hymnographie kommt vor allem im Triodion (der Tessarakoste, ab dem Montag der ersten Fastenwoche bis zum Lazarussamstag) zum Ausdruck, aber auch in der Sammlung der Oktoechos (dem Acht-Wochen-Zyklus in den acht Tönen), den Menaia (dem Zyklus der unbeweglichen Feste vom 1. September bis zum 31. August) und im Pentekostarion (vom Ostersonntag bis zum Sonntag nach Pfingsten, dem Allerheiligen-Sonntag).

Betrachten wir zuerst die Fastenzeit, so stehen uns zwei Arten von Quellen zur Verfügung, die Auskunft über den Einfluss des Theodoros auf die Hymnographie geben. Es sind dies:

1. liturgische Typika, in denen der Ablauf der Offizien mit ihren Psalmen, Lesungen und Hymnen für das gesamte Kirchenjahr aufgezeichnet sind, und

2. die Sticheraria, musikliturgische Bücher, welche die Gesänge des großen Chores zur Stichologie (zu den Psalmversen) enthalten.

Unter den liturgischen Typika sind vor allem das Typikon von Messina, das auf die Tradition des 12. Jahrhunderts ${ }^{6}$ zurückgeht, und das Typikon

${ }^{4}$ Ein Kanon ist die Einheit von neun bzw. acht hymnischen Oden, die in ihrem Text auf die neun bzw. acht biblischen Oden Bezug nehmen. Jede Ode besteht aus einem Heirmos, einer Musterstrophe, der mehrere Troparia in Melodie und Metrum nachgebildet werden. Jede Ode hat eine eigene Melodie. Meist sind die Heirmoi eines Kanons durch eine Akrostichis verbunden. Die Kanonesdichtung geht vor allem auf die Palästinenser Andreas von Kreta, Johannes von Damaskos und Kosmas von Jerusalem zurück.

${ }_{5}^{5}$ Stichera sind Hymnen, meist in rhythmischer Prosa, die zu den sechs bis acht letzten Psalmversen der Stichologie im Morgen- und im Abendoffizium gesungen werden. Ihre Texte sind unabhängig von den Psalmversen, zwischen die diese Gesänge eingeschoben werden. Man unterscheidet Stichera idiomela, die meist nur einmal im Kirchenjahr gesungen werden, Stichera automela, die anderen Gesängen als Musterstrophen dienen, und Stichera prosomoia, die den Automela metrisch und musikalisch nachgebildet werden.

${ }^{6}$ M. Arranz, Le typicon du monastère du Saint-Sauveur à Messine: Codex Messinensis gr. 115 (OCA 185). Rom 1969. Arranz datiert die Handschrift in das Jahr 1131. Leroy versucht nachzuweisen, dass dieses Typikon erst in der 2 . Hälfte des 13 . Jahrhunderts geschrieben wurde: J. Leroy, La date du Typicon de Messine et de ses manuscrits. BollGrott 24 (1970). 
des Euergetis-Klosters von Konstantinopel aus dem 11.-12. Jahrhundert ${ }^{7}$ von Bedeutung. Als Ergänzung dient das Triodion der römischen Ausgabe ${ }^{8}$. Das Typikon der Hagia Sophia von Konstantinopel, von dem die früheste Überlieferung aus dem 9.-10. Jahrhundert stammt, weist, bis auf einige Troparia, keinen Bezug zur Hymnodie der Klostertypika auf.

Die Zeit des Fastens, der Buße und der Vorbereitung auf die Kreuzigung und die Auferstehung Christi verlangt auch in der Hymnologie nach einer Einschränkung der melodischen Vielfalt. Die Kanones werden von acht bzw. neun Oden auf drei Oden reduziert, viele Gesänge haben keine eigene Melodie, sondern werden Modellstrophen nachgebildet.

Eng verflochten mit der Hymnologie des Theodoros Studites für die Fastenzeit ist die seines Bruders Joseph. Ab dem Montag der ersten Fas-

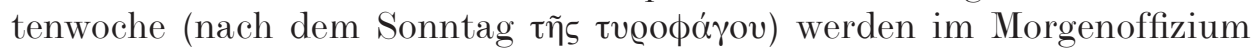
(Orthros) zur Stichologie zwei Kathismata des Triodion von Joseph von Thessalonike und ein Kathisma des Theodoros gesungen ${ }^{9}$. Unmittelbar nach dem Psalm 50 im Morgenoffizium kommen je ein Triodion (ein auf drei Heirmoi verkürzter Kanon) des Joseph und des Theodoros zum Vor$\operatorname{trag}^{10}$. Zuerst werden der erste Heirmos des Triodion des Joseph und des Theodoros gesungen, dann folgen der zweite und der dritte Heirmos. So sind die hymnischen Oden der beiden Brüder ineinander verschachtelt; es kann aber durchaus vorkommen, dass die beiden Triodia nicht dem gleichen Modus (Echos) angehören ${ }^{11}$. Die Triodia von Montag bis Freitag beginnen mit der ersten, zweiten, dritten, vierten bzw. fünften Ode, diesen folgen stets die achte und die neunte Ode. Die Heirmoi und Troparia dieser Triodia sind metrisch und melodisch den Oden einzelner Kanones nachgebildet; sie sind daher Prosomoia ${ }^{12}$. Die Melodien dieser Heirmoi sind in den liturgischen Büchern kaum greifbar, da auch ihre Modellstrophen (Automela) vorwiegend der oralen Tradition angehören.

\footnotetext{
7 A. DmitrievskiJ, Opisanie liturgičeskich rukopisej chranjaščichsja v bibliotekach pravoslavnago Vostoka I. Kiev 1895, 256-656.

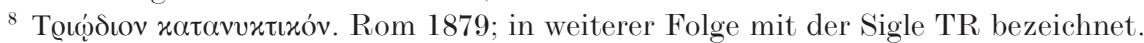

${ }^{9}$ Kathismata sind im allgemeinen in den Musikhandschriften äußerst selten zu finden. Ihre Melodien dürften Modellstrophen (Automela) nachgebildet sein, die das gesamte Kirchenjahr Verwendung fanden und deshalb als bekannt vorausgesetzt wurden.

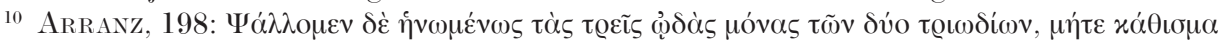

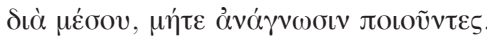

$11 \mathrm{Vgl}$. TR 282.

${ }^{12}$ In TR steht immer vor der Angabe des Triodion: Пஹò ऽ tó, diesem folgt das Incipit eines Kanons. Im Typikon von Messina und im Typikon des Euergetis-Klosters fehlen sowohl die Incipits der Modellkanones als auch der Triodia von Joseph und Theodoros.
} 
Anders ist die musikalische Überlieferung der Stichera prosomoia des Theodoros und des Joseph für das Abendoffizium (Esperinos) der Fastenzeit. Bereits in den Sticheraria des 11. Jahrhunderts sind die Prosomoia des Theodoros mit Notation aufgezeichnet. Zu erwähnen sind hier, unter anderen, der Codex Athos Vatopedi 1488 von der Mitte des 11. Jahrhunderts und der Codex Ochrid $53^{13}$ aus der zweiten Hälfte des 11. Jahrhunderts. In beiden Handschriften sind die Prosomoia in einem adiastematischen paläobyzantinischen Notationsstadium ${ }^{14}$ überliefert: in Vatopedi 1488 in Chartres-Notation, in Ochrid 53 in Coislin-Notation ${ }^{15}$. Hier sind die Stichera prosomoia des Theodoros zwischen den Idiomela für die Wochentage der Fastenzeit mit den Incipits der Modellstrophen aufgezeichnet, in Vatopedi 1488 hingegen sind sie für die sechs Wochen der Fastenzeit ge-

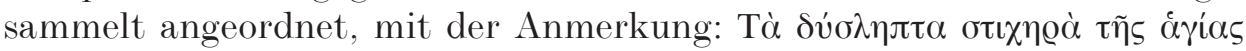

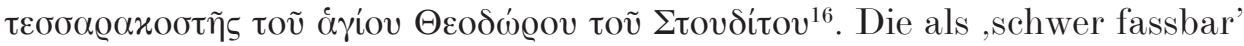
bezeichneten Prosomoia weisen darauf hin, dass ihre Melodien nicht Automela nachgebildet wurden, die in der mündlichen Praxis verankert waren, sondern dass für diese Gesänge vor allem Idiomela als Musterstrophen dienten, die im Sticherarion aufgezeichnet sind. Theodoros zog für diese Prosomoia vor allem Hymnen jener Heiligen heran, die durch ihr Martyrium, ihr asketisches Leben oder ihr bedingungsloses Eintreten für den Glauben zum Vorbild für die Kirche wurden. Die Stichera prosomoia des Joseph sind erst in den Quellen jüngeren Datums zu finden, so z.B. im Codex Vindob. theol. gr. 181 aus dem Jahr 1221 ${ }^{17}$. Hier sind die Prosomoia in ihrer Gesamtheit zusammengefasst, allerdings ohne Nennung einer Musterstrophe. Im Abendoffizium wurden zu Psalm 140 je zwei Prosomoia des Joseph und eines des Theodoros gesungen. Die zwei Prosomoia des Joseph haben meist die gleiche Melodie, sie sind aber trotzdem beide in den Codices jüngeren Datums mit Notation aufgezeichnet. H. Husmann weist nach,

${ }^{13}$ Naroden Muzej, Ochrid. Beschreibung der Handschrift von V. Mošıs, Rakopisi na Narodniot muzej vo Ochrid. Zbornik na trudovi (Ochrid 1961), $218 f$.

${ }^{14}$ Diese frühen Notationssysteme zeigen nur den ungefähren melodischen Verlauf mit Hilfe der Notenzeichen an. Erst ab der zweiten Hälfte des 12. Jahrhunderts, mit der Einführung der sog. mittelbyzantinischen Notation, können die einzelnen Gesänge entziffert werden.

${ }^{15}$ Eine kurze Beschreibung dieser Codices findet sich bei C. Floros, Universale Neumenkunde 1. Kassel 1970, 55f. und 57.

${ }^{16}$ E. Follieri - O. Strunk, Triodium Athoum. Codex Monasterii Vatopedii 1488 phototypice depictus (MMB 9). Kopenhagen 1975, f. $213 \mathrm{v}$.

17 C. Høeg, H.J.W. Tillyard, E. Wellesz, Sticherarium (MMB 1). Kopenhagen 1935, f.311v-320v. Eine Transkription der Prosomoia des Triodion unternahm H.J.W. Tillyard, The Hymns of the Octoechus (MMB Transcripta 5, 2). Kopenhagen 1949, $1-58$. 
dass Joseph Studites für dreißig Prosomoia-Paare sechzehn Musterstrophen verwendet, die alle Automela sind ${ }^{18}$. Eine Aufzeichnung dieser Gesänge schien daher den Schreibern der ältesten musikalischen Handschriften nicht erforderlich zu sein.

Theodoros Studites nahm nicht nur durch seine Hymnographie Einfluss auf die liturgische Gestaltung der Tessarakoste; auch die von ihm verfassten Katechesen wurden fester Bestandteil der Horen während der klösterlichen Fastenzeit. Laut dem Euergetis-Typikon werden ab dem Sonntag

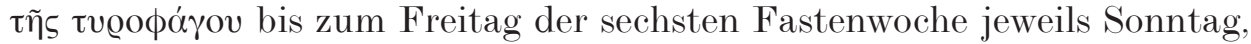
Mittwoch und Freitag die Katechesen des Theodoros in der ersten Hore gelesen. Das Typikon von Messina schreibt ab dem Montag der ersten Fastenwoche bis Freitag der sechsten Woche für jeden Wochentag, ausgenommen Mittwoch, die Lesung der Katechesen des Theodoros in der dritten Hore vor, beginnend mit der ersten Katechese des ersten Buches ${ }^{19}$. Von

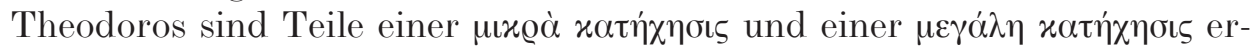
halten $^{20}$. Es handelt sich hier vorwiegend um asketische Schriften, die vor allem die Pflichten des Mönchslebens zum Gegenstand haben und auf die Ideale christlicher Vollkommenheit hinweisen. Diese Katechesen sind zum Teil in einer sehr poetischen Form verfasst, so dass viele Passagen an hymnische Texte erinnern. Zwei Beispiele sollen dies verdeutlichen:

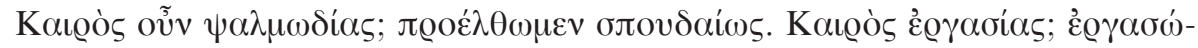

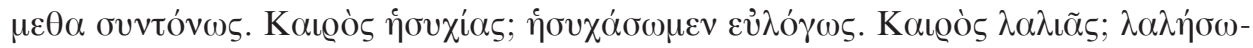
$\mu \varepsilon v \stackrel{3}{\varepsilon} \mu \mu \varepsilon \lambda \tilde{\omega} \varsigma^{21} \ldots$

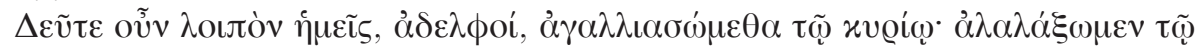

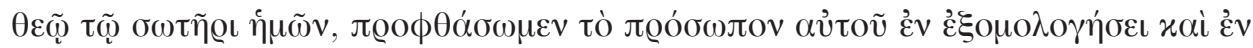

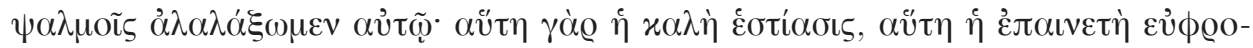

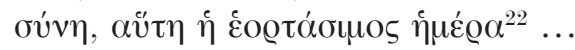

${ }^{18}$ H. Husmann, Die Prosomoia der Großen Fastenzeit. Archiv für Musikwissenschaft 29 (1972) 216-231; N. Schidlovsky, The Notated Lenten Prosomoia in the Byzantine and Slavic Traditions. Princeton 1983.

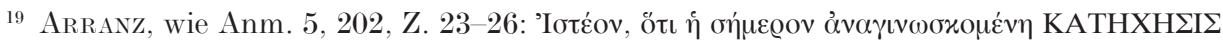

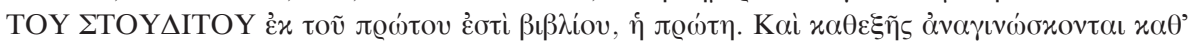

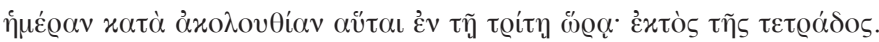

${ }^{20}$ J. Cozza-LuzI, S. Theodori Studitae sermones parvae catecheseos (Nova Patrum Bibliotheca 9,1). Rom 1888; E. Auvray, Theodori parva katechesis. Paris 1891; A. Papadopou-

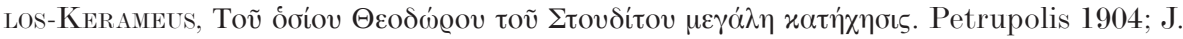
Cozza-Luzi, S. Theodori Studitae sermones reliqui magnae catecheseos (Nova Patrum Bibliotheca 10.1). Rom 1905.

${ }^{21}$ Dieser Text ist der Kleinen Katechese 61, für den Freitag der dritten Fastenwoche, entnommen. Vgl. Cozza-Luzi (Nova Patrum Bibliotheca 9,1), 145.

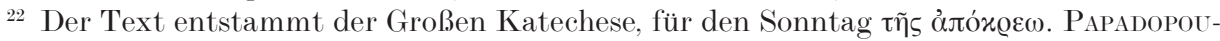
Los-Kerameus, wie Anm. 19, $646 \mathrm{f}$. 
Die Reden der Kleinen Katechese dürften früher verfasst worden sein,

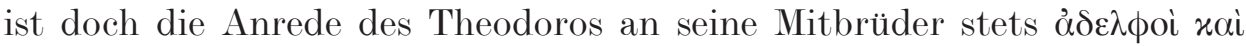

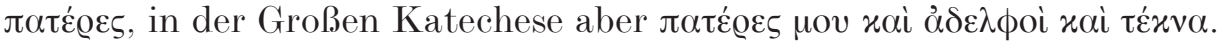

Einen bedeutenden Beitrag zur Sammlung der Oktoechos leistete Theodoros Studites mit seinen Anabathmoi, den Antiphona der Oktoechos ${ }^{23}$. Sie werden im Morgenoffizium, vor dem Morgenevangelium, im jeweiligen Modus der Woche, alternierend von zwei Chören gesungen. Diese Anabathmoi bestehen aus einer Einheit von drei bzw. vier kleinen Antiphonen der Psalmen 119 bis 130 und 132, der psalmi graduum. Für jeden der acht Echoi (Modi) der acht Sonntage der Oktoechos ist eine solche Einheit bestimmt. Sie finden sich in den ältesten musikliturgischen Sticheraria, wie z.B. in Athos Laura $\Gamma 67$ vom Beginn des 11. Jahrhunderts, in Athos Vatopedi 1488 von der Mitte des 11. Jahrhunderts und in Ochrid 53 aus der zweiten Hälfte des 11. Jahrhunderts ${ }^{24}$. Diese Sticheraria überliefern die Anabathmoi gesammelt. Sie enthalten nicht nur die Troparia ${ }^{25}$, welche zu den Psalmversen gesungen werden, sondern auch die Psalmstichoi selbst. Diese sind allerdings ohne Neumierung. Die Sticheraria des 13./14. Jahrhunderts überliefern die Troparia fast immer ohne Psalmverse, innerhalb des Zyklus der Auferstehungsgesänge, nach dem jeweiligen Echos geordnet. Vindob. theol. gr. $181^{26}$ fügt jedem Antiphonon einen Psalmvers bei, der jedoch den Psalmen 6 und 36 entnommen ist. Dies lässt vermuten, dass im Lauf der Jahrhunderte der Gesang der Psalmverse zu den Troparia immer mehr reduziert wurde.

$\mathrm{Zu}$ den beiden ersten Troparia werden je zwei Psalmverse gesungen, jedes Troparion wird zweimal vorgetragen. Die dritte Strophe ist ein Triadikon, das von einer Doxologie eingerahmt ist. Es beginnt stets mit den

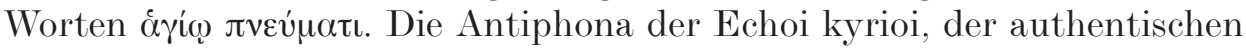
Modi, entnehmen ihre Verse den Psalmen 119 bis 130. Die Echoi plagioi, die plagalen Modi, beginnen wieder mit Psalm 119 und verwenden die glei-

${ }^{23}$ Ein Kommentar zu den Anabathmoi des Theodoros aus dem 14. Jahrhundert stammt

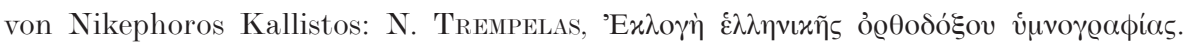
Athen 1949, เ $\varepsilon^{\prime}$, เ $\eta^{\prime}$. Einen ausführlichen Artikel über die Anabathmoi verfasste O. Strunk, The Antiphons of the Oktoehos, in: Journal of the American Musicological Society 13 (1960) 50-67 (Nachdruck in: O. Strunk, Essays on Music in the Byzantine World. New York 1977, 165-190). Transkribiert wurden die Anabathmoi von H.J.W. Tillyard, The Hymns of the Octoechus (MMB Transcripta 3, 1). Kopenhagen 1940, $145-183$.

${ }^{24} \mathrm{Vgl}$. Anm. 15.

25 Troparia sind kurze poetische Hymnen, die meist in einem syllabischen Stil verfasst wurden; d.h. pro Silbe werden ein oder zwei Noten gesungen.

${ }^{26} \mathrm{Vgl}$. Anm. 17. 
chen Stichoi wie die authentischen Modi. Nur der 4. plagale Echos fügt ein viertes Antiphonon an, mit Versen aus dem Psalm $132^{27}$.

Die Troparia der Anabathmoi stellen in ihrem engen Bezug zu den Psalmstichoi eine Besonderheit in der byzantinischen Hymnographie dar. Die ersten beiden Strophen jedes Antiphonon beginnen mit einem wörtlichen Zitat aus dem jeweiligen Psalmvers. Der weitere Text stellt eine Paraphrase dieses Stichos dar. Die Troparia der plagalen Echoi beginnen ebenfalls mit dem gleichen Psalmtext, setzen jedoch in anderer Weise die Paraphrase fort. In immer neuer Variation schuf Theodoros den Text für diese Strophen. Es entstand somit eine völlig neue Form der Hymnologie. Jene Antiphona, die älter sind als die Anabathmoi und dem palästinensischen Raum entstammen, enthalten Troparia, die zwar in ihrem Text auf das jeweilige Fest Bezug nehmen, jedoch keine Verbindung zu den sie umrahmenden Psalmversen herstellen. Wir finden diese Form der Antiphona in der Weihnachts- und Epiphaneia-Paramonie und im Großen Leidensgottesdienst am Karfreitag, aufgezeichnet in den Sticheraria.

Was die Melodie der Troparia des Theodoros betrifft, so wird am Beginn jeder Strophe der Psalmtext mit einer einfachen psalmodischen Kadenz eingeleitet, d.h. die letzten vier Silben werden, ohne Rücksicht auf ihren Akzent, automatisch an die letzten vier musikalischen Elemente angepasst. Der poetische Text, der dem Psalmzitat folgt, setzt sich in den für den jeweiligen Echos charakteristischen melodischen Formeln fort.

Diese Form der Tropierung stellt für die frühe Periode der byzantinischen Hymnographie ein exzeptionelles Beispiel dar. Erst ab dem 14. Jahrhundert finden wir in den spätbyzantinischen Handschriften, den Akolouthiai, im Rahmen der Stichologie der Psalmen 134 und 135, der sog. Polyeleoi, eine ähnliche Form. Die Troparia beginnen mit einem Stichos im Psalmton, dem ein kurzer Hymnus, meist ein Gesang auf die Theotokos, in Fünfzehnsilbern folgt ${ }^{28}$. Diese dem Psalmton folgenden Melodien gehören allerdings nicht, wie jene des Theodoros, dem syllabischen Stil an. Sie sind melodisch reich ornamentiert und entsprechen der kalophonischen Tradition der spätbyzantinischen Zeit ${ }^{29}$. Der Großteil dieser Tropierungen stammt von Johannes Kukuzeles.

27 Die Verse des Psalms 132 sind bestimmten Heiligenfesten vorbehalten. Vgl. STRunk, Essays, 167, Anm. 9.

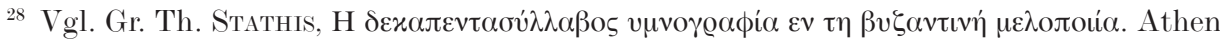
$1977,197-202$.

29 Beispiele für eine Tropierung des Polyeleos finden sich im Codex Vindob. theol. gr. 185, f. $128 \mathrm{v}-129 \mathrm{v}$; im Codex Vindob. suppl. gr. 110, f. 326r; im Codex Vindob. phil. gr. 194, f. $64 \mathrm{r}-65 \mathrm{r}$. 
Einen interessanten Hinweis auf die Kompositionstechnik des Theodoros Studites und seines Bruders Joseph gibt der Traktat des sog. Hagiopolites, einer musiktheoretischen Schrift, die in Jerusalem entstanden ist und deren Abfassungszeit auf das 12. Jahrhundert zurückgeht. Hier findet sich die Feststellung, dass zwar die Gesänge des Johannes von Damaskos und des Kosmas von Maiuma im 2. Echos mesos, dem Nenano und im 4. Echos

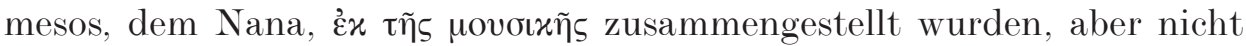
jene der beiden Brüder ${ }^{30}$. Untersucht man die Melodien der zitierten Gesänge des Johannes und des Kosmas, so ist festzustellen, dass ihre Melodien die charakteristischen symphonen Intervalle der antiken Musiktheorie aufweisen: Oktav, Quint und Quart. Die Behauptung, dass die Melodien des Theodoros und des Joseph nicht der Musiktheorie entsprächen, konnte zum Teil bestätigt werden ${ }^{31}$. Untersucht man die Gesänge des Theodoros, so kann im zweiten und dritten Troparion des dritten Antiphonon der Anabathmoi, die in das Nenano modulieren, festgestellt werden, dass der Klangraum nur von $e-d^{\prime}$ bzw. von $e-c^{\prime}$ reicht, also nur über eine Septim bzw. eine Sext geht. Im vierten Antiphonon modulieren das zweite und dritte Troparion vom 4. plagalen Echos in das Nana; der Klangraum umfasst nur $g-e^{\prime}$ bzw. $g-f^{\prime}$, also eine Sext bzw. eine Septim; die charakteristischen Quinten bzw. Quarten sind jedoch feststellbar. Aus dem Zitat des ,Hagiopolites ist jedenfalls zu schließen, dass für die Meloden des 7.-9. Jahrhunderts Kriterien der klassisch-griechischen Musiktheorie von Bedeutung waren.

Die hymnographische Tätigkeit des Theodoros erstreckte sich nicht nur auf Gesänge für den Zyklus der Tessarakoste und der Oktoechos, sondern umfasste darüber hinaus das gesamte Kirchenjahr mit seinen beweglichen und unbeweglichen Festen ${ }^{32}$.

Eine Reihe von Kontakia ist unter dem Namen des Theodoros überliefert $^{33}$. Es handelt sich hierbei vor allem um Prosomoia. Auffallend ist, dass viele der mit ihrem Text überlieferten Kontakia vor allem Kirchenvätern gewidmet sind, die sich für den orthodoxen Glauben einsetzten. Die Kontakia sind von unterschiedlicher Länge; sie haben zwischen vier und vier-

${ }^{30}$ Vgl. J. RaAsted, The Hagiopolites. A Byzantine Treatise on Musical Theory. Cahiers de l'Institut du Moyen-Âge Grec et Latin 45 (Kopenhagen 1983), § 2, 1-22; G. Wolfram - Ch. Hannick, Die Erotapokriseis des Pseudo-Johannes Damaskenos zum Kirchengesang (Corpus Scriptorum de Re Musica 5). Wien 1997, Z. 727-741, Kommentar 138f.

${ }_{31}$ Vgl. Wolfram - Hannick, Die Erotapokriseis des Pseudo-Johannes Damaskenos, Kommentar 138-145.

${ }^{32}$ Eine bibliographische Zusammenfassung gibt J. Szöverffry, A Guide to Byzantine Hymnography. A Classified Bibliography of Texts and Studies, 2. Brookline, Mass. and Leyden 1979, 31-33.

${ }^{33}$ Vgl. J.B. Pitra, Analecta Sacra Spicilegio Solesmensi parata 1. Paris 1876, 336-380. 
zehn Strophen, die nur zum Teil durch eine Akrostichis zusammengefasst sind.

Neben Stichera prosomoia für die Fastenzeit verfasste Theodoros auch Stichera idiomela ${ }^{34}$, die vor allem einzelnen Heiligen gewidmet sind. Nur ganz vereinzelt sind solche Idiomela in den musikliturgischen Handschriften zu finden ${ }^{35}$.

Die Kanones des Theodoros sind durchwegs Prosomoia. Ihre Protypa sind die Kanones der palästinensischen Mönche Andreas von Kreta, Johannes von Damaskos und Kosmas von Maiuma. Als Katabasia, als abschlieBenden Heirmos jeder Ode, benützt Theodoros stets den Heirmos seines Modellkanons. Was die Kanones des Theodoros von jenen der Palästinenser unterscheidet, ist, dass es sich nicht mehr in jeder Ode um eine Paraphrasierung der biblischen Ode handelt, sondern dass sich das Thema eines Kanons in allen Oden fortsetzt.

In den verschiedenen hymnographischen Schöpfungen des Theodoros kommt einerseits zum Ausdruck, dass er um eine Übernahme der palästinensischen Tradition in den Raum Konstantinopel bemüht war, dass er aber auch an einer liturgischen Erneuerung interessiert war. Inwieweit noch vor der endgültigen Wiederherstellung der Bilderverehrung am Konzil von Konstantinopel (843) eine liturgische Erneuerung wirksam wurde, bleibt fraglich. Es darf jedoch angenommen werden, dass der Einfluss des Theodoros an den reformierten liturgischen Büchern sehr groß ist.

${ }^{34}$ W. Christ - M. Paranikas, Anthologia graeca carminum Christianorum. Leipzig 1871 (Nachdruck Hildesheim 1963), $101 \mathrm{f}$.

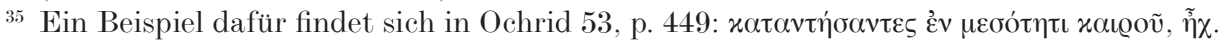
$\pi \lambda . \delta^{\prime}$. 
